\title{
LOCALIZATION AT INJECTIVES IN COMPLETE CATEGORIES
}

\author{
J. LAMBEK AND B. A. RATTRAY
}

Abstract. We consider a complete category $\&$. For each object $I$ of $\mathscr{A}$ we define a functor $Q: \mathscr{A} \rightarrow \mathscr{A}$ and obtain a necessary and sufficient condition on $I$ for $Q$, after restricting its codomain, to become a reflector of $\alpha$ onto the limit closure of $I$. In particular, this condition is satisfied if $I$ is injective in $\mathscr{S}$ with regard to equalizers. Among the special cases of such reflectors are: the reflector onto torsion-free divisible objects associated to an injective $I$ in $\operatorname{Mod} R$; the Samuel compactification of a uniform space; the Stone-Cech compactification.

We give a second description of $Q$ in terms of a triple on sets. If $I$ is injective and the functor $Q$ is equivalent to the identity then, under a few extra conditions on $\mathscr{L}, \mathscr{A}^{\text {op }}$ is triplable over sets with regard to the functor taking $A$ to $\mathscr{A}(A, I)$.

We recall some notation and definitions. We write $\mathscr{A}(A, B)$ or just $(A, B)$ for the set of all maps from $A$ to $B$ in $\mathscr{A} . \mathscr{S}$ denotes the category of sets. The limit closure of an object $I$ of $\mathscr{A}$ is the smallest full replete subcategory of $\mathscr{A}$ closed under limits and containing $I$. I is injective with regard to the map $f: A \rightarrow B$ if $\mathscr{A}(f, I): \mathscr{A}(B, I) \rightarrow \mathscr{A}(A, I)$ is a surjection. We call $I$ injective in $\mathscr{A}$ if it is injective with regard to all regular monomorphisms in $\mathscr{A}$. A regular monomorphism is a map which happens to be an equalizer.

The object $I$ determines functors

$$
\mathscr{A} \stackrel{(-, I)}{\longrightarrow} \mathscr{S}^{(0)} \stackrel{I^{(-)}}{\longrightarrow} \mathscr{A},
$$

where $(-, I)$ is a left adjoint of $I^{(-)}$, in view of the natural isomorphism

$$
\mathscr{S}^{\mathrm{op}}(\mathscr{A}(A, I), X)=\mathscr{S}(X, \mathscr{A}(A, I)) \cong \mathscr{A}\left(A, I^{\mathrm{X}}\right) .
$$

Thus the composition $S=I^{(-, I)}$ is part of a triple (standard construction) $(S, \eta, \mu)$ on $\mathscr{A}$ (see [9]). For future reference we describe $\eta(A): A \rightarrow S(A)$ and also $S(f)$ for any map $f: A \rightarrow B$.

Received by the editors June 7, 1972 and, in revised form, January 2, 1973.

AMS (MOS) subject classifications (1970). Primary 18A40; Secondary 18C15, $18 \mathrm{E} 35,18 \mathrm{E} 40,54 \mathrm{E} 15$.

Key words and phrases. Localization, reflective subcategory, injective, triple, torsion theory. 
Let $u^{*}$ denote the map $A \rightarrow I^{X}$ corresponding to $u: X \rightarrow(A, I)$, defined by the formula $\forall_{x \in X} \pi_{x} u^{*}=u(x)$, where $\pi_{x}$ is the canonical projection $I^{X} \rightarrow I$. Then $\eta(A)=1_{(A, I)}^{*}$, that is,

$$
\forall_{g \in(A, I)} \pi_{g} \eta(A)=g .
$$

The map $S(f)=I^{(f, I)}: I^{(A, I)} \rightarrow I^{(B . I)}$ is given thus

$$
\forall_{f \in(A, B)} \forall_{h \in(B, I)} \pi_{h} S(f)=\pi_{h f} .
$$

Following Fakir [2], we define the functor $Q: \mathscr{A} \rightarrow \mathscr{A}$ as the equalizer

$$
Q \stackrel{\kappa}{\longrightarrow} S \underset{S \eta}{\stackrel{\eta S}{\longrightarrow}} S^{2} \text {. }
$$

Fakir showed that $Q$ is part of a triple $\left(Q, \eta_{1}, \mu_{1}\right)$ and that, if $S(\kappa(A))$ is mono for each object $A$, then $Q$ is idempotent.

Let Fix $Q$ be the full subcategory of $\mathscr{A}$ consisting of all those objects $A$ for which $\eta_{1}(A): A \rightarrow Q(A)$ is an isomorphism. Since, $Q(A)$ is only defined up to isomorphism, we can assume that each such $\eta_{1}(A)$ is the identity map of $A$. Then $Q$ is idempotent if and only if, by restriction of the codomain to the image, it induces a reflector $\mathscr{A} \rightarrow$ Fix $Q$. The reflection map from $A$ into Fix $Q$ is then $\eta_{1}(A): A \rightarrow Q(A)$, which is defined by the condition $\kappa(A) \eta_{1}(A)=\eta(A)$.

Before stating our main result, we require two lemmas.

LEMMA 1. $\quad \kappa(A): Q(A) \rightarrow S(A)$ is the joint equalizer of all pairs of maps $S(A) \rightrightarrows I$ which coequalize $\eta(A): A \rightarrow S(A)$.

Proof. Consider any map $u: S(A) \rightarrow I$. Then, by $(*), \pi_{u} \eta S(\stackrel{\AA}{A})=u$, and by $\left({ }^{* *}\right), \pi_{u} S \eta(A)=\pi_{u \eta(A)}$. Thus $\kappa(A)$ equalizes all pairs of maps $\left(u, \pi_{u \eta(A)}\right)$. Now let $v: S(A) \rightarrow I$ be such that $u \eta(A)=v \eta(A)$. Then $\kappa(A)$ equalizes $(u, v)$, since

$$
u \kappa(A)=\pi_{u \eta(A)} \kappa(A)=\pi_{v \eta(A)} \kappa(A)=v \kappa(A) .
$$

Conversely, any map which equalizes all $(u, v)$ such that $u \eta(A)=v \eta(A)$ equalizes $\left(u, \pi_{u \eta(A)}\right)$ in particular, since $\pi_{u \eta(A)} \eta(A)=u \eta(A)$ by $\left({ }^{*}\right)$. Hence it equalizes $\eta S(A)$ and $S \eta(A)$.

\section{Lemma 2. $I \in$ Fix $Q$.}

Proof. By $(*), \pi_{1_{I}} \eta(I)=1_{I}$, hence $\eta(I)$ is the equalizer of the pair of maps $\left(\eta(I) \pi_{1}, 1_{S(I)}\right)$. Thus $\eta(I)$ is the joint equalizer of all pairs of maps $I^{(I, I)} \rightarrow I^{(I, I)}$ which coequalize $\eta(I)$, and therefore the joint equalizer of all pairs of maps $I^{(I, I)} \underset{\rightarrow}{\rightarrow}$ which coequalize $\eta(I)$. In view of Lemma 1 , 
$\kappa(I)$ is this same equalizer. Hence the unique map $\eta_{1}(I): I \rightarrow Q(I)$ such that $\kappa(I) \eta_{1}(I)=\eta(I)$ is an isomorphism, and so $I$ is in Fix $Q .{ }^{1}$

THEOREM. The following statements are equivalent.

(a) I is injective with regard to $\kappa(A)$ for each object $A$ in $\mathscr{A}$.

(b) $Q$ is idempotent, i.e. becomes a reflector $\mathscr{A} \rightarrow$ Fix $Q$ when its codomain is restricted to its image.

(c) Fix $Q$ is the limit closure of $I$.

PRoOF. (b) and (c) are clearly equivalent, since

$$
I \in \text { Fix } Q \subset \text { image of } Q \subset \text { limit closure of } I
$$

and a reflective subcategory is limit closed.

(a) $\Rightarrow$ (b). We note that if $I$ is injective with regard to a map $f$ then $(f, I)$ is a surjection, that is, a regular mono of $\mathscr{S}^{\text {op }}$. Now $I^{(-)}$, being a right adjoint, preserves limits, so $S(f)=I^{(f, I)}$ is a regular mono of $\mathscr{A}$. Thus it follows from condition (a) that $S(\kappa(A))$ is mono for each $A$. Fakir's result (Proposition 3 of [2]) then shows that $Q$ is idempotent.

(b) $\Rightarrow$ (a). We have $A \rightarrow_{\eta_{1}(A)} Q(A) \rightarrow_{\kappa(A)} S(A)$ with $\kappa(A) \eta_{1}(A)=\eta(A)$. Now every map $u: A \rightarrow I$ can be extended to $\pi_{u}: S(A) \rightarrow I$, since $\pi_{u} \eta(A)=u$. Given any map $v: Q(A) \rightarrow I$, we let $u=v \eta_{1}(A)$. Then $\pi_{u} \kappa(A) \eta_{1}(A)=u=$ $v \eta_{1}(A)$. Since $\eta_{1}(A)$ is a reflection map of $A$ into Fix $Q$, it follows from Lemma 2 that $\pi_{u} \kappa(A)=v$. Thus $I$ is injective with regard to $\kappa(A)$.

This completes the proof of the theorem.

Definition. We shall call the object $I$ of $A \kappa$-injective if it satisfies the equivalent conditions of the theorem. We call $Q$ the localization functor determined by $I$.

COROLlaRY. If I is injective with regard to all equalizers of pairs of maps $I^{X} \rightarrow I^{Y}$ then $Q$ is (after restricting its codomain) a reflector onto the limit closure of $I$, which is Fix $Q$. If I is injective in $\mathscr{A}$ then $Q$ takes regular monos of $\mathscr{A}$ to regular monos of Fix $Q$.

Proof. The first statement is obvious. As for the second, let $I$ be injective in $\mathscr{A}$ and $f: A \rightarrow B$ a regular mono of $\mathscr{A}$. We already know that $S(f)$ is a regular mono of $\mathscr{A}$ which lies in Fix $Q$. Since Fix $Q$ is the limit closure of the injective $I$, Theorem 1 of [11] shows that $S(f)$ is a regular mono of Fix $Q$. Now $\kappa(A): Q(A) \rightarrow S(A)$ is also a regular mono of Fix $Q$ and it is easily seen that the composition of two regular monos of Fix $Q$ is a regular mono of Fix $Q$ (since an object $A$ is in Fix $Q$ iff there is an equalizer diagram $\left.A \rightarrow I^{n} \rightrightarrows I^{m}\right)$. Thus $\kappa(B) Q(f)=S(f) \kappa(A)$ is a regular

${ }^{1}$ The referee has pointed out that Lemma 2 can also be proved directly by observing that $\pi_{1}$ and $S\left(\pi_{1}\right)$ make $I \rightarrow S(I) \rightarrow S^{2}(I)$ into a split equalizer diagram. 
mono of Fix $Q$. Since $\kappa(B)$ is mono in Fix $Q, Q(f)$ is also a regular mono of Fix $Q$.

We remark that if $\mathscr{A}$ is well powered then, by the special adjoint functor theorem, the limit closure of any object $I$ is a reflective subcategory of $\mathscr{A}$.

We shall now consider a number of examples. Further examples in the categories of partially ordered sets, lattices, etc. are being studied by $\mathrm{B}$. Ballinger.

EXAMPLE 1. Take $\mathscr{A}=\operatorname{Mod} R$, where $R$ is an associative ring with unity, and let $I$ be any injective right $R$-module. We claim that $Q(A)$ is then the usual localization of $A$, also called the module of quotients of $A$, with respect to the torsion theory determined by $I$ (see [7]).

To prove this, let us provisionally denote the module of quotients by $Q^{\prime}(A)$. Then $Q^{\prime}(A)$ is divisible (with respect to $I$ ), in the terminology of [7] and may be regarded as a submodule of $S(A)$, since the kernel of $\eta(A)$ is the torsion submodule of $A$. Now $S(A)$, being a product of copies of $I$, is torsion-free (with respect to $I$ ), hence $S(A) / Q^{\prime}(A)$ is torsion-free. Since $Q^{\prime}(A) / \operatorname{Im} \eta(A)$ is torsion, it is the torsion submodule of $S(A) / \operatorname{Im} \eta(A)$. Thus $s \in Q^{\prime}(A)$ if and only if

that is,

$$
\forall_{u: S(A) \rightarrow I}(u \eta(A)=0 \Rightarrow u(s)=0),
$$

$$
\forall_{v, w: S(A) \rightarrow I}(v \eta(A)=w \eta(A) \Rightarrow v(s)=w(s)) .
$$

Therefore $Q^{\prime}(A)$ is the joint equalizer of all pairs of maps which coequalize $\eta(A)$. Hence, by Lemma $1, Q^{\prime}(A)=Q(A)$, as was to be proved.

EXAMPLE 2. Take $\mathscr{A}$ to be the category of topological spaces and let $I$ be the unit interval $[0,1] . I$ is not injective, but it is $\kappa$-injective. It is easily seen that $Q(A)$ is the closure in $S(A)$ of the image of $\eta(A)$. Thus the construction of $Q(A)$ is the familiar construction of the Stone-Čech compactification $\beta(A)$, as described by Cech [1]. Condition (a) in this example is simply a special case of Tietze's theorem, since $S(A)=I^{(A . I)}$ is normal and $Q(A)$ is a closed subspace.

Of course, there is no reason for $\beta$ to preserve regular monos, as $I$ is not injective (with regard to all regular monos) in $\mathscr{A} .^{2}$

EXAMPLE 3. Take $\mathscr{A}$ to be the category of uniform spaces (not necessarily Hausdorff) and let $I=[0,1]$. Regular monomorphisms in $\mathscr{A}$ are easily seen to be the same as subspace inclusions. It is known that $I$ is injective with regard to subspace inclusions (see [6]).

The reflector $Q$ is the Samuel compactification (see [12] or [5]), that is, Fix $Q$ consists of all compact Hausdorff uniform spaces.

\footnotetext{
${ }^{2}$ George Reynolds has observed that one can similarly obtain the real compactification of $A$ by taking $I$ to be the real line.
} 
To see this, we note that $Q(A)$ is easily shown to be the closure in $S(A)$ of the image of $\eta(A)$. Now $S(A)=I^{(A, I)}$ is compact, and therefore so is the closed subspace $Q(A)$. (We observe that the forgetful functor from uniform to topological spaces preserves products.) Conversely, assume that $A$ is compact Hausdorff, and recall that all continuous mappings from a compact Hausdorff uniform space are uniformly continuous. Then $Q(A)$ is the Stone-Čech compactification of $A$, and hence $\eta_{1}(A)$ is an isomorphism.

There are many other injectives in $\mathscr{A}$. For example, if $M$ is any metrizable uniform space then $(M, I)$ with the obvious metric is injective [5]. It is not clear what the associated category Fix $Q$ is. It certainly contains only complete spaces. It seems unlikely that there is any $M$ for which Fix $Q$ contains all complete spaces, that is, such that $Q(A)$ is the Hausdorff completion of $A .^{3}$

EXAMPLE 4 . Let $\mathscr{A}$ be the category of presheaves on a small category $\mathscr{X}$, that is, the category of all functors $\mathscr{X}^{\mathrm{op}} \rightarrow \mathscr{S}$. Suppose that $\mathscr{X}$ is equipped with a Grothendieck topology, then one can construct a huge injective $I$ whose limit closure is the category of sheaves for the given topology. For any presheaf $A, Q(A)$ is then the associated sheaf.

The story is somewhat different when $\mathscr{A}$ is an elementary topos in the sense of Lawvere and Tierney. Since $\mathscr{A}$ is not necessarily complete, our construction of the triple $S$ does not work. However, an analogous construction does work, and we shall sketch it briefly.

Let $I$ be any injective in $\mathscr{A}$. Since $\mathscr{A}$ is a cartesian closed category, we can obtain a triple $S$ on $\mathscr{A}$ from the selfadjoint functor $\mathscr{A} \rightarrow \mathscr{A}^{\text {op }}$ taking $A$ to $I^{A}$, with $S(A)=I^{A}$. Fakir's construction applied to $S$ then gives an idempotent triple $Q$. However, Fix $Q$ is not the limit closure of $I$; it must also be closed under internal powers. It turns out that $Q$ preserves all finite limits.

The work of Lawvere and Tierney suggests which injectives $I$ one should single out for consideration: Let $j$ be any Heyting endomorphism of the subobject classifier $\Omega$, then take $I=\Omega_{j}$, the retract of $\Omega$ determined by $j$. Fix $Q$ will then be the category of $j$-sheaves. (For definitions see the discussion of the work of Lawvere and Tierney in [3].)

We plan to elaborate the details of this example in a sequel to the present paper.

We shall give another interpretation of the localization functor $Q$

\footnotetext{
${ }^{3}$ Indeed, George Reynolds has observed that the completion functor in uniform spaces cannot be obtained by our method from a single uniform space $I$, because any such space has a cardinal number associated with it which is preserved by products and subspace formation, namely the smallest infinite cardinal such that every uniform cover has a refinement less than it.
} 
with the help of the Eilenberg-Moore category of a triple. It will be convenient here to replace the complete category $\mathscr{A}$ by its opposite, the cocomplete category $\mathscr{B}=\mathscr{A}^{\text {op }}$. We consider an object $P$ of $\mathscr{B}$ which is $\kappa$-projective, that is, $\kappa$-injective as an object of $\mathscr{A}$.

The functor $U=(P,-): \mathscr{B} \rightarrow \mathscr{S}$ has a left adjoint $F$, where $F(X)=\sum_{x \in X} P$, let us say with adjunction $\eta:$ id $\rightarrow U F$ and coadjunction $\varepsilon: F U \rightarrow$ id. The triple $(U F, \eta, U \varepsilon F)$ on $\mathscr{S}$ gives rise to the Eilenberg-Moore category $\mathscr{S}^{U F}$, whose objects are certain pairs $(X, \xi)$, where $\xi: U F(X) \rightarrow X$ in $\mathscr{S}$. One studies the so-called comparison functor $K: \mathscr{B} \rightarrow \mathscr{S}^{U F}$ given by

$$
K(B)=(U(B), U \varepsilon(B)), \quad K(b)=U(b) .
$$

This has a left adjoint $M$ (see [9, p. 151, Exercise 5]), where $M(X, \xi)$ is the coequalizing object of the pair

$$
F U F(X) \underset{\varepsilon F(X)}{\stackrel{F(\xi)}{\longrightarrow}} F(X) .
$$

A simple calculation shows the following:

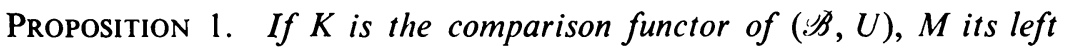
adjoint, and $Q$ the localization functor on $\mathscr{B}^{\text {op }}$, then $M K=Q$.

We may also call $Q$ the colocalization functor on $\mathscr{H}$.

We consider an interesting special case.

EXAmple 5. Take $\mathscr{B}=\operatorname{Mod} R$ and let $P$ be any finitely generated $\kappa$-projective right $R$-module. Let $E$ be the ring $(P, P)$, then $(P,-)$ may be considered as a functor $\mathscr{B} \rightarrow \operatorname{Mod} E$ with left adjoint $(-) \otimes_{E} P$. Since $P$ is finitely generated, $(P,-)$ takes sums in $\mathscr{B}$ to sums in Mod $E$. Let $U_{E}$ denote the forgetful functor $\operatorname{Mod} E \rightarrow \mathscr{S}$. Then the triple $U F$ is given by

$$
U F(X)=U_{E}\left(P, \sum_{X^{\prime}} P\right) \cong U_{E}\left(\sum_{X^{*}} E\right) .
$$

Now this is the triple associated to $\operatorname{Mod} E$, so $\mathscr{S}^{U} \boldsymbol{F}=\operatorname{Mod} E$. The functor $K$ is clearly $(P,-)$, hence $M=(-) \otimes_{E} P$, and we have

$$
Q(B)=(P, B) \otimes_{E} P .
$$

This formula actually holds in a more general situation. Let $\mathscr{B}$ be any cocomplete abelian category and $P$ any $\boldsymbol{\kappa}$-projective object which is small in the sense that $(P,-): \mathscr{B} \rightarrow \operatorname{Mod} E$ preserves sums.

The smallness of $P$ will follow easily if it is assumed to be finitely generated in the sense of $[4]$, that is, that $(P,-)$ preserves directed colimits of monomorphisms. In this definition it makes no difference whether $(P,-)$ is considered as a functor into $\operatorname{Mod} E$ or into $\mathscr{S}^{4}$

\footnotetext{
${ }^{4} \mathrm{We}$ are indebted to the referee for criticizing the original discussion of this example, in which the condition that $\boldsymbol{P}$ be finitely generated had been overlooked.
} 
Proposition 2. Let $Q$ be the colocalization functor determined by the $\kappa$-projective object $P$ of the cocomplete category $\mathscr{B}$. Then the following conditions are equivalent.

(1) $Q$ is canonically isomorphic to the identity, that is, $\eta_{1}$ is a natural isomorphism.

(2) $P$ is a regular generator in the sense that for each object $B$ of $\mathscr{B}$ there is a regular epi from some multiple of $P$ to $B$.

(3) For each object $B$ of $\mathscr{B}$ there is a coequalizer diagram $m P \rightrightarrows n \rightarrow B \rightarrow B$, for some cardinal numbers $m$ and $n$.

(4) $\mathscr{B}$ is the colimit closure of $P$.

Proof. The implication (4) $\Rightarrow(1)$ is an immediate consequence of our theorem. For, since $P$ is $\kappa$-projective, the theorem tells us that the colimit of $P$ is Fix $Q$, and by (4) this is $\mathscr{B}$, so that (1) holds.

The implications $(1) \Rightarrow(2) \Rightarrow(3) \Rightarrow(4)$ hold even without the assumption that $P$ is $\kappa$-projective. The first and last implications are clear; we shall prove that $(2) \Rightarrow(3)$. Assume (2) and let $B$ be any object of $\mathscr{B}$. Then there

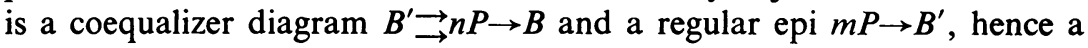
coequalizer diagram $m P \rightarrow \vec{\rightarrow} P \rightarrow B$.

Clearly (1) implies that $P$ is $\kappa$-projective. Hence (1) asserts that $P$ is a $\kappa$-projective regular generator.

We present the following variant of Linton's theorem (see $[8$, p. 88]).

PROPOSITION 3. Let $P$ be an object of the category $\mathscr{B}$ and $U=\mathscr{B}(P,-)$, then $(\mathscr{B}, U)$ is varietal (triplable) if and only if

(1) $\mathscr{B}$ is cocomplete and has kernel pairs,

(2) $P$ is a projective regular generator,

(3) every equivalence relation in $\mathscr{B}$ is a kernel pair.

A pair of maps $R \rightrightarrows \rightarrow A$ in $\mathscr{B}$ is here called an equivalence relation if $\mathscr{B}(B, R) \rightrightarrows \mathscr{B}(B, A)$ is an equivalence relation in $\mathscr{S}$ for every object $B$ of $\mathscr{B} .^{5}$

Proof. Necessity of conditions. (1) and (3) are well-known properties of varietal categories, and (2) follows from Proposition 1.

Sufficiency of conditions. We verify Linton's conditions FIT ([8, p. 88]). We know that $U$ has a left adjoint and that $(F I T)_{0}$ holds, that is, $\mathscr{B}$ has kernel pairs and coequalizers. $(F I T)_{1}$ says that $\pi: A \rightarrow B$ in $\mathscr{B}$ is a regular epi if and only if $U(\pi)$ is a surjection.

Since $P$ is projective, $U$ takes regular epis to surjections. Conversely, if $U(\pi)$ is a surjection, then $F U(\pi)$ is a regular epi, since $F$ preserves

\footnotetext{
${ }^{5}$ Both M. Barr and C. Mulvey have informed us that they have obtained essentially the same version of Linton's theorem.
} 
limits. Now consider the following commutative diagram:

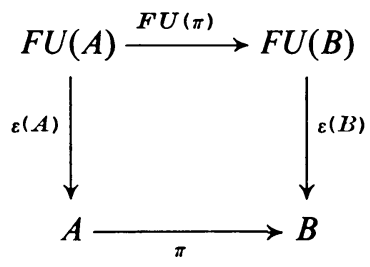

Since $Q \cong$ id canonically, $\varepsilon(A)$ and $\varepsilon(B)$ are regular epis. Now the composition $\varepsilon(B) F U(\pi)$ of two regular epis is a regular epi, since $\mathscr{B}$ is the limit closure of the projective $P$ (see [11, Lemma 2.2]). Since $\pi \varepsilon(A)$ is a regular epi and $\varepsilon(A)$ is epi, $\pi$ is a regular epi.

$(F I T)_{2}$ says that $p_{1}, p_{2}: R \rightrightarrows A$ is a kernel pair in $\mathscr{B}$ if and only if $U\left(p_{1}\right)$, $U\left(p_{2}\right): U(R) \rightrightarrows U(A)$ is a kernel pair in $\mathscr{S}$. The "only if" part is obvious, since $U$ is representable. Consider the full subcategory $\mathscr{C}$ of $\mathscr{B}$ consisting of those objects $B$ for which the representable functor $(B,-)$ takes $\left(p_{1}, p_{2}\right)$ into a kernel pair. $\mathscr{C}$ contains $\boldsymbol{P}$ and is obviously replete. It is easily seen to be closed under colimits, and since $\mathscr{B}$ is the colimit closure of $P, \mathscr{C}=\mathscr{B}$.

COROLlaRY 1. Let $\mathscr{A}$ be a complete Abelian category with an injective cogenerator I. Then $\mathscr{A}^{\mathrm{p}}$ is varietal with respect to the functor $\mathscr{A}(-, I)$.

ProOf. $\mathscr{B}=\mathscr{A}^{\mathrm{op}}$ satisfies the conditions of Proposition 3 with $P=I$. Indeed, conditions (1) and (2) are obvious. To prove (3) we observe that in Mod $R$ every equivalence relation is a kernel pair. By Mitchell's embedding theorem, the same is true in any Abelian category, hence in the opposite of an Abelian category.

EXAMPLE 6. The opposite of any Grothendieck category $\mathscr{A}$ is varietal, as $\mathscr{A}$ contains an injective cogenerator. Oberst [10] has also described $\mathscr{A}^{\text {op }}$ as a concrete category with the forgetful functor $\mathscr{A}(-, I)$. However, the structure he defines involves topology and is not obviously varietal.

COROLLARY 2. Let $Q$ be the colocalization functor associated with the projective object $P$ of the cocomplete category $\mathscr{B}$. Assume $\mathscr{B}$ has kernel pairs and all equivalence relations in the category Fix $Q$ are kernel pairs. Then Fix $Q$ is varietal with respect to the functor $(P,-)$ : Fix $Q \rightarrow \mathscr{S}$.

Proof. Fix $Q$ satisfies conditions (1) to (3) of Proposition 3.

Example 6 could also have been deduced from Corollary 2 by means of the Gabriel-Popescu theorem.

EXAMPLE 7. Let $\mathscr{A}$ be the category of all set-valued sheaves with respect to a Grothendieck topology. Then $\mathscr{A}^{\text {op }}$ is varietal.

A proof will be given in a sequel to this paper. 


\section{REFERENCES}

1. E. Čech, On bicompact spaces, Ann. of Math. 38 (1937), 823-844.

2. S. Fakir, Monade idempotente associée à une monade, C.R. Acad. Sci. Paris Sér. A-B 270 (1970), A99-A101. MR 41 \#1828.

3. P. Freyd, Aspects of topoi, Bull. Austral. Math. Soc. 7 (1972), 1-76.

4. P. Gabriel and F. Ulmer, Lokal präsentierbare Kategorien, Lecture Notes in Math., vol. 221, Springer-Verlag, Berlin and New York, 1971.

5. J. R. Isbell, Uniform spaces, Math. Surveys, no. 12, Amer. Math. Soc., Providence, R.I., 1964. MR 30 \#561.

6. M. Katetov, On real-valued functions in topological spaces, Fund. Math. 38 (1951), 85-91. MR 14, 304.

7. J. Lambek, Torsion theories, additive semantics, and rings of quotients, Lecture Notes in Math., vol. 177, Springer-Verlag, Berlin and New York, 1971. MR 44 \#1685.

8. F. Linton, Some aspects of equational categories, Proc. Conf. Categorical Algebra (La Jolla, Calif., 1965), Springer, New York, 1966, pp. 84-94. MR 35 \#233.

9. S. Mac Lane, Categories for the working mathematician, Springer-Verlag, Berlin and New York, 1971.

10. U. Oberst, Duality theory for Grothendieck categories and linearly compact rings, J. Algebra 15 (1970), 473-542.

11. B. Rattray, Non-additive torsion theories, Manuscripta Math. (to appear).

12. P. Samuel, Ultrafilters and compactifications of uniform spaces, Trans. Amer. Math. Soc. 64 (1948) 100-132. MR 10, 54.

Department of Mathematics, McGill University, Montreal, Quebec, Canada 